\title{
sciendo
}

Research Article

(C) 2019 Nasser B. Ebrahim and Madhu S. Atteraya. This is an open access article licensed under the Creative Commons Attribution-NonCommercial-NoDerivs License

(http://creativecommons.org/licenses/by-nc-nd/3.0/).

\section{Women's Household Decision-Making and Intimate Partner Violence in Ethiopia}

\author{
Nasser B. Ebrahim, Ph.D. \\ Assistant Professor, Department of Public Health, \\ Keimyung University, 1095 Dalgubeol-Daero, Dalseo-Gu. \\ Daegu 42601, South Korea \\ Corresponding Author \\ Madhu S. Atteraya, Ph.D. \\ Assistant Professor, Department of Social Welfare, \\ Keimyung University, 1095 Dalgubeol-Daero, Dalseo-Gu, \\ Daegu 42601, South Korea
}

Doi: 10.2478/ajis-2019-0041

\begin{abstract}
Women's household decision-making, a reflection of interpersonal power dynamics in intimate relationships is assumed to play a central role in eliminating violence against women. Thus we sought to examine the association between women's household decision-making autonomy and the occurrence of intimate partner violence (IPV) among Ethiopian women. We used data gathered in the 2016 Ethiopia Demographic and Health Survey (EDHS-2016). We limited our study to ever-married women (aged: 15 49 years) who responded to the domestic violence questions ( $n=4,469)$. Sampling weights were applied and effects associated with complex survey design were accounted for. Overall, 24\%, 23.1\%, and $10.1 \%$ of women have experienced emotional abuse, physical violence, or sexual violence, respectively in their lifetimes. The relationship between demographic variables and IPV were inconsistent and mostly non-significant. We found significant association between decision-making autonomy and IPV variables. Women who made decisions jointly with their husbands/partners had lower risk of domestic violence as compared to women with low level of household decision-making autonomy. No significant difference between women in the low and high level of decision-making groups. Egalitarian family power structures may be beneficial toward reducing IPV and achieving gender equality in Ethiopia.
\end{abstract}

Keywords: Ethiopia, women, intimate partner violence, decision-making autonomy, empowerment

\section{Introduction}

Intimate partner violence (IPV) is a pervasive global public health problem with devastating health consequences affecting one in three women worldwide [World Health Organization (WHO), 2017]. It is defined as the infliction of psychological, physical, or sexual harm in the context of intimate relationships (WHO, 2012). Results from a large international study by the WHO (Garcia-Moreno, Jansen, Ellsberg, Hiese, \& Watts, 2006) showed a lifetime prevalence of 15 to $71 \%$ for physical or sexual violence among women from diversified cultures, locations, and settings. In Africa, nearly four in 10 partnered women experience IPV in their lifetime, the highest global prevalence (WHO, 2013). Women who are victims of IPV often face physical and/or mental health problems including serious bodily injury, chronic pain, and reproductive health problems (Campbell, 2002; WHO, 
2017). IPV is a common occurrence among Ethiopian women (Guruge et al., 2012; Semahegn \& Mengistie, 2015). A recent report based on a nationally representative sample showed a lifetime physical and/or sexual IPV rate of $28 \%$ [Central Statistical Agency (CSA) and ICF, 2016]. However, in rural regions of Ethiopia, a lifetime prevalence of $71 \%$ has been reported (Garcia-Moreno et al., 2006).

Violence against women is ingrained in many cultures and societies. This is reflected in both social and political structures that stem largely from gender inequalities, norms, and values that put women in a subordinate position-displayed by the traditional tolerance and acceptance of violence against women (Hunnicutt, 2009; WHO, 2010). Commonly cited factors associated with IPV include poverty, power inequality, relationship conflicts, alcohol use, and societal norms (Jewkes, 2002). For example, feminist theory attributes domestic violence in patriarchal societies to gender power imbalances and structural factors that limit women's participation in societal and economic activities. The resulting inequality in women's positions in societies and relationships engenders and perpetuates violence against women (Dobash, 1979; Yodanis, 2004). Empowering women and achieving gender equality appear to ameliorate IPV (Jewkes, 2002; Ebrahim \& Atteraya, 2018). Women's household decision-making, a reflection of interpersonal power dynamics in intimate relationships, has become an important focus for governments because it is assumed to play a central role in eliminating violence against women (United Nations, 2013). Hence, promoting equality and empowerment of women is one of the 17 Sustainable Development Goals of the United Nations (United Nations, 2015). Theories linking women's increased autonomy to lower rates of IPV assume that the former reinforces women's capacity to negotiate favorable outcomes in the face of lopsided power dynamics in patriarchal societies (Jewkes, 2002).

Women in many African countries, including Ethiopia, face tremendous challenges that emanate from gender inequality and power imbalance. For example, Ethiopia is ranked 115 of 144 countries in gender equality index calculated from women's economic participation, educational attainment, health \& survival, and political empowerment (World Economic Forum, 2017). While some improvements have been made in recent years, women in Ethiopia still face inequities and limited opportunities for development, education, and employment (United Nations, 2013). This eroded social position likely drives violence against women. Therefore, the purpose of our study was to understand the association between women's decision-making autonomy, as a measure of empowerment, and the occurrence of IPV among Ethiopian women. We found no prior studies that examined women's empowerment and domestic violence variables from nationally representative samples in the Ethiopian context and believe this work will fill a knowledge gap in this respect.

\section{Methods}

\subsection{Sample Design}

We used data collected in the 2016 Ethiopia Demographic and Health Survey (EDHS-2016) by the Ethiopian CSA. The goal of that survey was to provide vital health information at the national level. The 2016 EDHS survey was comprehensive in its scope and, for the first time, included women's experiences of emotional, physical, and sexual abuse (CSA and ICF, 2016).

A two-stage stratified cluster sampling method was used to select the survey sample. In the first stage, a total of 645 enumeration areas were identified and classified as either rural $(n=443)$ or urban $(n=202)$. In the second stage, 28 households per enumeration area were selected (CSA and ICF, 2016). The survey included responses collected face-to-face from 15,683 women. However, we limited our study to ever-married women (aged: 15 - 49 years) who responded to the domestic violence questions $(n=4,469)$. We applied sampling weights and accounted for any effects associated with the complex survey design.

\subsection{Measurements}

The dependent variables in this study were women's lifetime experience of emotional abuse, physical, or sexual violence from their current or most recent husband/partner. The lifetime 
experience of emotional abuse by women was estimated from responses to the following three questions: (1) "Have you ever been humiliated in front of others by your husband/partner?" (2) "Have you ever been threatened with harm by your husband/partner?" and (3) "Have you ever been insulted or made to feel bad by your husband/partner?" Answers to these questions were either "yes" or "no" and summative scores were generated by coding "yes" responses as 1 and "no" responses as 0 .

The lifetime experience of physical violence by women was estimated using six questions: (1) "Have you ever been pushed, shaken, or had something thrown at you by your husband/partner?" (2) "Have you ever been slapped by your husband/partner?" (3) "Have you ever been punched or hit with something harmful by your husband/partner?" (4) "Have you ever been kicked or dragged by your husband/partner?" (5) "Have you ever been strangled or burnt by your husband/partner?" and (6) "Have you ever been threatened with a knife, gun, or another weapon by your husband/partner?" Answers to each of these questions were "yes" or "no." Initially, the responses were aggregated and women who answered "yes" to any of these questions were considered to have experienced physical violence and coded as 1 . On the other hand, women who answered "no" to all of the items were considered to have not experienced physical violence and coded as 0 .

Women's lifetime experiences of sexual violence were estimated from the following three items: (1) "Have you ever been physically forced by your husband/partner into sex when you did not want to?" (2) "Have you ever been forced into other unwanted sexual acts by your husband/partner?" and (3) "Have you ever been forced with threats or in any other way to perform sexual acts you did not want?" Responses to these questions were "yes" or "no," and the same coding system as described above was used to group women based on their lifetime experience of sexual violence.

The main independent variable in this study was women's household decision-making autonomy. This factor was assessed by determining: (1) person who usually decides on respondent's healthcare; (2) person who usually decides on large household purchases; (3) person who usually decides on visits to family or relatives; and (4) person who usually decides on household finances. Answers to these questions were: (1) respondent alone, (2) respondent and husband/partner, (3) respondent and another person, (4) husband/partner alone, (5) someone else, and (6) other persons. Responses to the four questions were aggregated (range $4-22$, median = 8 ) and then used to categorize women into three levels of household decision-making autonomy. Women whose husbands/partners or someone else were making household decisions had low levels of decision-making autonomy, reflected by scores greater than eight. Scores of eight were reflective of medium levels of autonomy and these women shared household decisions with their husbands/partners. Women with scores less than eight were categorized as having high levels of decision-making autonomy, as they were largely responsible for making household decisions.

In addition, we included socio-demographic variables: respondent's age, residence (rural or urban), religion, wealth index (generated from household income, consumption, and expenditures), respondent's education level and employment status, and the husband/partner's education level and employment status.

\section{Data Analysis}

We performed all data analyses with the module for complex survey design in SPSS v25.0. Bivariate associations between IPV and the study variables were examined using the Chi-square test. The parameters of association between the IPV variables and independent variables were estimated using multivariate logistic regressions. We used a p-value of 0.05 to determine statistical significance.

\section{Results}

Overall, $24 \%, 23.1 \%$, and $10.1 \%$ of women have lifetime experienced emotional abuse, physical violence, and sexual violence, respectively. In the bivariate analyses, most demographic variables were significantly associated with the IPV variables (Table 1). Similarly, women's experiences with 
domestic violence significantly varied according to their level of decision-making autonomy (Table 1).

Significant associations between most of the demographic variables and IPV variables were inconsistent and mostly not significant in multivariate analyses (Table 2). The only variable that consistently associated with IPV variables across measures of domestic violence was women's household decision-making autonomy. The odds of women experiencing emotional abuse during their lifetime was $35 \%(\mathrm{OR}=0.65$; $\mathrm{Cl}$ : $0.48-0.89)$ less for women with medium levels of decisionmaking autonomy (women who share household decisions with their husband/partner) than women with low levels. Likewise, women with medium household decision-making autonomy were $48 \%$ $(\mathrm{OR}=0.52 ; \mathrm{Cl}: 0.38-0.72)$ and $38 \%(\mathrm{OR}=0.62 ; \mathrm{Cl}: 0.38-0.98)$ less likely to have lifetime experienced physical and sexual violence, respectively, than women with low household decisionmaking autonomy. No significant difference was observed in the occurrence of IPV among women in the low and high decision-making groups (Table 2).

\section{Discussion}

In contrast to previous studies on domestic violence against women that used proxy measures such as attitude toward IPV, this study is based on self-reported experiences of IPV among Ethiopian women from a nationally representative sample (CSA [Ethiopia] and ORC Macro, 2006; CSA [Ethiopia] and ORC Macro, 2012; CSA and ICF, 2016). Nearly, one-quarter of Ethiopian women reported experiencing emotional or physical abuse in their lifetime and one-tenth experienced sexual abuse. Our results concurred with a previous report from Ethiopia (CSA and ICF, 2016). The prevalence of IPV in Ethiopia, although substantial, is lower than the $37 \%$ average lifetime physical and/or sexual IPV reported for ever-partnered women in sub-Saharan Africa (WHO, 2013). In the multivariate analyses, the relationship between demographic variables and IPV were inconsistent and mostly non-significant; this suggests that socio-demographic variables were not central in predicting IPV in our study population and may also reflect the lack of variation in domestic violence experiences among Ethiopian households. Neither women's wealth, education level, nor employment status are protective against IPV in sub-Saharan Africa (Cools \& Kotsadam, 2017; González-Brenes, 2004). In contrast, women with fewer economic resources than their husbands/partners were reported to be at a greater risk of domestic violence in some Asian countries (Hindin \& Adair, 2002; Kerley \& Sirisunyaluck, 2011).

We found women's decision-making autonomy to be a significant predictor of IPV in our study population; however, the association was not linear and in agreement with earlier report (Jewkes, 2002). Unlike women who made joint decisions with their husbands/partners (medium decisionmaking autonomy), low or high decision-making autonomy was associated with higher risks of IPV. Women who made decisions jointly with their husband/partner, however, had the lowest risk of psychological, physical, or sexual violence. Our results are in agreement with numerous reports from a diverse population of women (Jewkes, 2002; Hindin \& Adair, 2002; Gage, 2004; Rahman, Nakamura, Seino, \& Kizuki, 2013; Svec \& Andic, 2018; Xu, Kerley, \& Sirisunyaluck, 2011).

In patriarchal societies like Ethiopia where male dominance over and violence against women are tolerated, women's control of household decisions may elevate their risk of experiencing violence (Hindin \& Adair, 2002; Kim \& Emery, 2003). This may be linked to the change in marital power dynamics being inconsistent with societal norms and this being perceived to undermine the male's position (Coleman \& Straus, 1986; Kim \& Emery, 2003). 
Table 1. Socio-demographic factors and women's decision-making autonomy in relation to domestic violence among ever married women

\begin{tabular}{|c|c|c|c|c|c|c|c|c|c|c|c|c|}
\hline \multirow{3}{*}{ Total } & \multicolumn{4}{|c|}{ Ever any emotional abuse } & \multicolumn{4}{|c|}{ Ever any physical abuse } & \multicolumn{4}{|c|}{ Ever any sexual abuse } \\
\hline & \multicolumn{4}{|c|}{$1071 / 4469(24 \%)$} & \multicolumn{4}{|c|}{$1034 / 4469(23.1 \%)$} & \multicolumn{4}{|c|}{$451 / 4469(10.1 \%)$} \\
\hline & Subtotal & $\begin{array}{c}\text { No } \\
\text { N (\%) }\end{array}$ & $\begin{array}{c}\text { Yes } \\
N(\%)\end{array}$ & $P$ & Subtotal & $\begin{array}{c}\text { No } \\
\mathrm{N}(\%)\end{array}$ & $\begin{array}{c}\text { Yes } \\
\text { N (\%) }\end{array}$ & $P$ & Subtotal & $\begin{array}{c}\text { No } \\
N(\%)\end{array}$ & $\begin{array}{c}\text { Yes } \\
\text { N (\%) }\end{array}$ & $P$ \\
\hline \multicolumn{13}{|l|}{ Age in years } \\
\hline $15-24$ & 958 & $744(77.7)$ & 214 & & 958 & $727(75.9)$ & $231(24.1)$ & & 958 & $873(91.2)$ & $85(8.8)$ & \\
\hline $25-34$ & 1882 & $1469(78.1)$ & ) $413(21.9)$ & .043 & 1882 & 1449 (77.0) & $433(23)$ & .88 & 1882 & 1685 (89.5) & $197(10.5)$ & .64 \\
\hline $35-49$ & 1629 & $1185(72.7)$ & $445(27.3)$ & & 1629 & $1258(77.2)$ & $371(22.8)$ & & 1629 & $1460(89.6)$ & $170(10.4)$ & \\
\hline \multicolumn{13}{|l|}{ Residence } \\
\hline Urban & 809 & $637(78.7)$ & $172(21.3)$ & & 809 & $664(82.1)$ & $145(17.9)$ & & 809 & $760(94.0)$ & $49(6.0)$ & 003 \\
\hline Rural & 3660 & $2761(75.4)$ & $899(24.6)$ & .22 & 3660 & $2771(75.7)$ & $890(24.3)$ & .01 & 3660 & $3258(89.0)$ & $403(11.0)$ & .003 \\
\hline \multicolumn{13}{|l|}{ Religion } \\
\hline Christians & 2947 & $2204(74.8)$ & $742(25.2)$ & & 2947 & $2270(77.0)$ & $677(23.0)$ & & 2947 & $2656(90.1)$ & $291(9.9)$ & \\
\hline Muslims & 1448 & $1148(79.3)$ & $299(20.7)$ & .015 & 1448 & $1120(77.3)$ & $328(22.7)$ & .14 & 1448 & $1292(89.3)$ & $156(10.7)$ & .70 \\
\hline Traditional/others & 75 & $45(60.3)$ & $30(39.7)$ & & 75 & $45(60.7)$ & $29(39.3)$ & & 75 & $70(93.3)$ & $5(6.9)$ & \\
\hline \multicolumn{13}{|l|}{ usehold wealth } \\
\hline Poor & 1699 & $1276(75.1)$ & $423(24.9)$ & & 1699 & $1301(76.5)$ & 399 & & 1699 & $1484(87.3)$ & $215(12.7)$ & \\
\hline Middle & 932 & $685(73.5)$ & $247(26.5)$ & .23 & 933 & $689(73.9)$ & $243(26.1)$ & .26 & 933 & $821(88.0)$ & $112(11.0)$ & .0001 \\
\hline Rich & 1838 & $1437(78.2)$ & $400(21.8)$ & & 1838 & $1445(78.6)$ & $392(21.4)$ & & 1838 & $1713(93.2)$ & $124(6.8)$ & \\
\hline \multicolumn{13}{|c|}{ Educational Level (women) } \\
\hline No education & 2725 & $2020(74.1)$ & 705 (25.9) & & 2725 & $2061(75.6)$ & $664(24.4)$ & & 2725 & 2409 (88.4) & $316(11.6)$ & \\
\hline Primary & 1236 & $937(75.8)$ & $299(24.2)$ & 001 & 1236 & $939(75.9)$ & $297(241)$ & & 1236 & $1125(91.0)$ & $111(9.0)$ & \\
\hline Secondary & 312 & $260(83.3)$ & $52(16.7)$ & & 312 & $256(81.9)$ & $56(18.1)$ & .008 & 312 & $295(94.5)$ & $17(5.5)^{\prime}$ & .046 \\
\hline Higher & 196 & $181(92.2)$ & $15(7.8)$ & & 196 & $180(91.5)$ & $17(8.5)$ & & 196 & $189(96.1)$ & $8(3.9)$ & \\
\hline \multicolumn{13}{|c|}{ Employment status (women) } \\
\hline Unemployed & 2895 & $2196(75.9)$ & $698(24.1)$ & & 2895 & $2228(77.0)$ & $667(23.0)$ & & 2895 & $2565(88.6)$ & $330(11.4)$ & \\
\hline Employed & 1574 & $1201(76.3)$ & $373(23.7)$ & .86 & 1754 & $1207(76.7)$ & $367(23.3)$ & .90 & 1574 & $1453(92.3)$ & $121(7.7)$ & .009 \\
\hline \multicolumn{13}{|c|}{ Husband/partner educational level } \\
\hline No education & 1807 & $1353(74.8)$ & $455(25.2)$ & & 1807 & $1377(76.2)$ & $430(23.8)$ & & 1807 & $1613(89.2)$ & $195(10.8)$ & \\
\hline Primary & 1397 & 1069 (76.5) & $328(23.5)$ & & 1397 & 1074 (76.9) & $322(23.1)$ & & 1397 & $1258(90.0)$ & 139 (10.0) & .064 \\
\hline Secondary & 361 & $301(83.3)$ & 60 & .002 & 361 & $306(84.8)$ & $55(15.2)$ & 000 & 361 & $339(93.9)$ & $22(6.1)$ & \\
\hline Higher & 299 & $266(88.9)$ & $33(11.1)$ & & 299 & $273(91.2)$ & $26(8.8)$ & & 299 & $288(96.2)$ & $11(3.8)$ & \\
\hline \multicolumn{13}{|l|}{ Husband/partner } \\
\hline Unemployed & 284 & $214(75.2)$ & $71(24.8)$ & 65 & 284 & $220(77.6)$ & $64(22.4)$ & 83 & 284 & $250(88.0)$ & $34(12.0)$ & 48 \\
\hline Employed & 3537 & $2731(77.2)$ & $806(22.8)$ & .00 & 3537 & $2775(78.4)$ & $762(21.6)$ & .00 & 3537 & $3204(90.6)$ & $333(9.4)$ & .40 \\
\hline \multicolumn{13}{|c|}{ Women decision-making } \\
\hline Low† & 614 & $462(75.2)$ & $152(24.8)$ & 001 & 614 & $451(73.5)$ & $163(26.5)$ & 003 & 614 & $549(89.5)$ & $65(10.5)$ & 29 \\
\hline Medium $\dagger+$ & 1970 & $1597(81.0)$ & $374(19.0)$ & .001 & 1971 & $1620(82.2)$ & $350(17.8)$ & .003 & 1971 & 1808 (91.7) & $163(8.3)$ & .29 \\
\hline High $\dagger+\dagger$ & 1285 & $936(72.8)$ & $349(27.2)$ & & 1285 & $973(75.7)$ & $312(24.3)$ & & 1285 & $1150(89.5)$ & 135 (10.5) & \\
\hline $\begin{array}{l}\text { P Probability of } \mathrm{s} \\
\text { counts have beer } \\
\dagger \text { Husbands/part } \\
\dagger † \text { Women/husb } \\
\dagger \dagger \dagger \text { To large exte }\end{array}$ & $\begin{array}{l}\text { tions }(P \\
\text { else wer } \\
\text { hare ho }\end{array}$ & $\begin{array}{l}\text { Pearson's } \mathrm{Cr} \\
\text { re making de } \\
\text { usehold dec }\end{array}$ & $\begin{array}{l}\text { ecisions } \\
\text { cisions }\end{array}$ & & e subtot & & & & & & & \\
\hline
\end{tabular}

Table 2: Adjusted odd-ratios (OR) and 95\% confidence $(95 \% \mathrm{Cl})$ : IPV by risk factors

\begin{tabular}{|c|c|c|c|c|c|c|}
\hline & \multicolumn{2}{|c|}{ Ever any emotional abus } & \multicolumn{2}{|c|}{ Ever any physical abuse } & \multicolumn{2}{|c|}{ Ever any sexual abuse } \\
\hline & OR & $96 \% \mathrm{Cl}$ & OR & $95 \% \mathrm{Cl}$ & OR & $95 \% \mathrm{Cl}$ \\
\hline \multicolumn{7}{|l|}{ Age in years } \\
\hline $15-24$ & 1 & & 1 & & 1 & \\
\hline $25-34$ & 1.08 & $.78-1.51$ & 1.09 & $.78-1.52$ & 1.27 & $.77-2.1$ \\
\hline $35-49$ & 1.50 & $1.08-1.09$ & 1.13 & $.79-1.60$ & 1.28 & $.77-2.16$ \\
\hline \multicolumn{7}{|l|}{ Residence } \\
\hline Rural & 1 & & 1 & & 1 & \\
\hline Urban & .92 & $.60-1.42$ & $.59^{*}$ & $.38-.93$ & .54 & $.19-1.50$ \\
\hline \multicolumn{7}{|l|}{ Religion } \\
\hline Christians & 1 & & 1 & & 1 & \\
\hline Muslims & $.71^{*}$ & $.53-.95$ & .99 & $.73-1.35$ & .95 & $.64-1.39$ \\
\hline Traditional/others & $1.97^{*}$ & $1.09-3.60$ & $2.4^{*}$ & $1.17-4.94$ & .60 & $.20-1.82$ \\
\hline
\end{tabular}




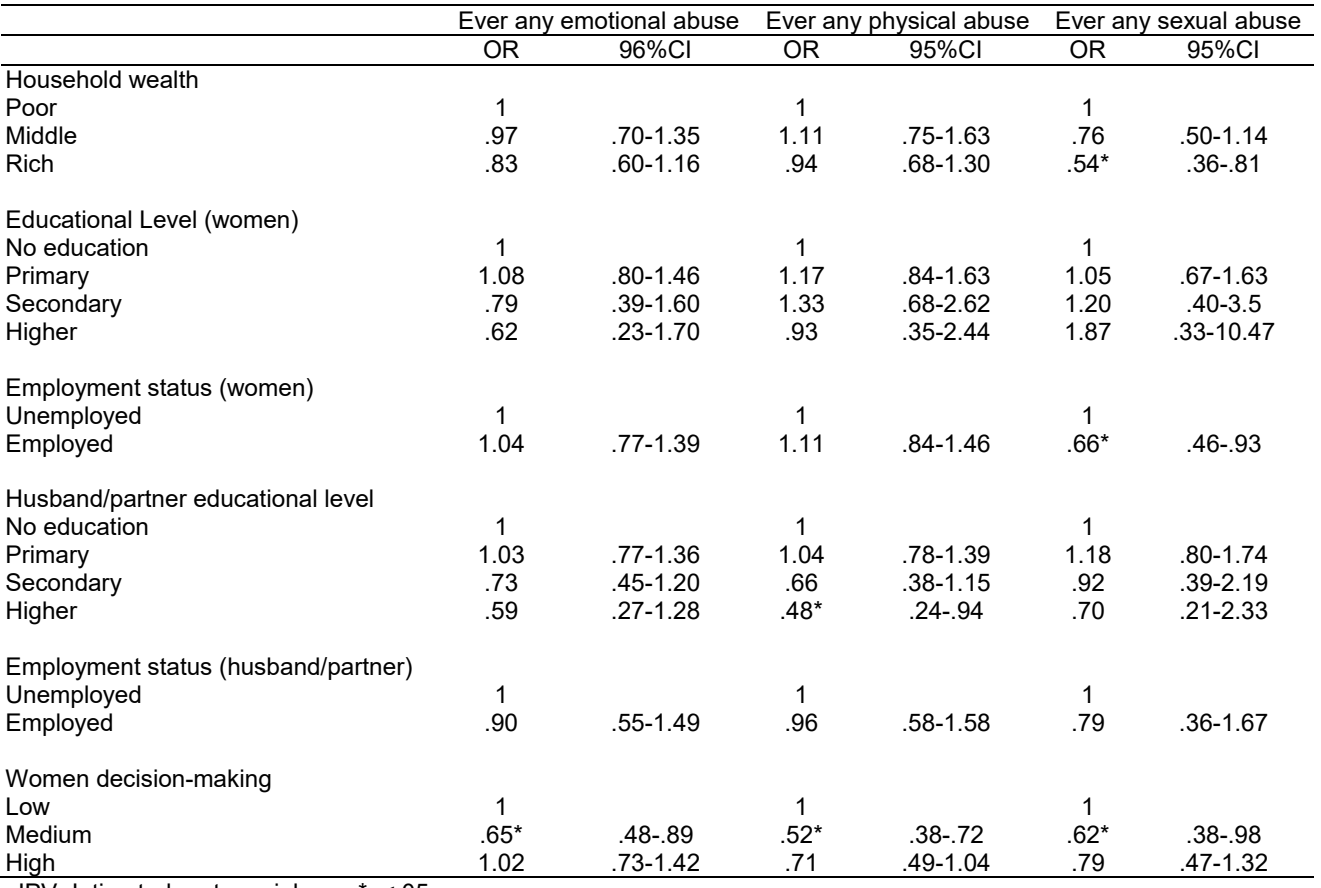

IPV $=$ Intimated partner violence ${ }^{*} p<.05$

Similarly, low decision-making autonomy was also associated with a higher risk of domestic violence. A male-dominated marital power structure has been reported to be highly associated with marital conflict and husband-to-wife violence (Kim \& Emery, 2003). Moreover, women who are socially and economically dependent on men are less likely to negotiate better treatment or abandon abusive relationships and thus more likely to tolerate violence (Kim, et al., 2007). In this study, women who reported making joint household decisions with their husbands/partners had the lowest risk of domestic violence. Coleman \& Straus (1986) provided the first evidence from the American population and found that the lowest risk of conflict and domestic violence was among equalitarian (egalitarian) couples in household decision-making. Kim \& Emery (2003) replicated Coleman \& Straus's study in the Korean population and also encountered similar results. Our results support that the egalitarian approach (shared household decision-making) seems to reduce IPV and contributes to achieving greater gender equality in Ethiopia. Nonetheless, the causes of violence against women are complex and involve the interplay between individual, relationship, community, and societal factors (WHO, 2002). Moreover, across Africa, including Ethiopia, empowered women are more likely to use modern contraceptives, skilled birth attendants, prenatal care, and HIV prevention services (Dugass, 2005; Ewerling et al., 2017; Taddesse, Teklie, Yizew, \& Gebreselassie, 2013). Thus, social and economic policies that empower women and encourage egalitarian decision-making may help toward reducing violence against women and improving the well-being of women in Ethiopia.

\section{Conclusions}

IPV is a common phenomenon among Ethiopian women. Women's household decision-making autonomy is a significant predictor of IPV. Women who engage in joint household decision-making with their husbands/partners have the lowest risk for experiencing domestic violence; therefore, egalitarian household power structures may be beneficial toward reducing IPV and achieving gender equality in Ethiopia. Fostering social and economic policies geared toward balancing women's positions within the household can potentially serve to improve women's well-being. 


\section{Limitations}

The study has some limitations. Because of the cross-sectional nature of the data, we report associations. Also, measurements were self-reported, hence there is a possibility of underestimation of intimate partner violence occurrence. Despite the limitations, the results are generalizable to the study population.

\section{References}

Campbell, J. C. (2002). Health consequences of intimate partner violence. The lancet, 359, 1331-1336. DOI: 10.1016/S0140-6736(02)08336-8.

Central Statistical Agency (CSA) [Ethiopia] and ICF. 2016. Ethiopia Demographic and Health Survey 2016. Addis Ababa, Ethiopia, and Rockville, Maryland, USA: CSA and ICF. https://dhsprogram.com/pubs/pdf/FR328/FR328.pdf.

Coleman, D. H., \& Straus, M. A. (1986). Marital power, conflict, and violence in a nationally representative sample of American couples. Violence and victims, 1(2), 141.

Cools, S., \& Kotsadam, A. (2017). Resources and intimate partner violence in Sub-Saharan Africa. World Development, 95, 211-230.https://doi.org/10.1016/j.worlddev.2017.02.027.

Dobash R. E, Dobash R.P. (1979) Violence Against Wives. New York: Free Press; 1979.

Dugassa, B. F. (2005). Women's rights and health: The case of Oromo women in Ethiopia. Health care for women international, 26 (2), 149-169. DOI: 10.1080/07399330590905594

Ebrahim, N. B., \& Atteraya, M. S. (2018). Women's Decision-Making Autonomy and their Attitude towards WifeBeating: Findings from the 2011 Ethiopia's Demographic and Health Survey. Journal of immigrant and minority health, 20(3), 603-611. DOI: 10.1007/s10903-017-0592-6.

Ewerling, F., Lynch, J. W., Victora, C. G., van Eerdewijk, A., Tyszler, M., \& Barros, A. J. (2017). The SWPER index for women's empowerment in Africa: development and validation of an index based on survey data. The Lancet Global Health, 5(9), e916-e923. DOI: https://doi.org/10.1016/S2214-109X(17)30292-9•

Garcia-Moreno, C., Jansen, H. A., Ellsberg, M., Heise, L., \& Watts, C. H. (2006). Prevalence of intimate partner violence: findings from the WHO multi-country study on women's health and domestic violence. The lancet, 368 (9543), 1260-1269. DOI: 10.1016/S0140-6736(06)69523-8.

González-Brenes, M. (2004). Domestic violence and household decision-making: evidence from East Africa. University of California, Berkeley. Retrieved from: http://www.scnet.ucla.edu/polisci/wgape/papers/7SGonzalez. pdf.

Guruge, S. (2012). Intimate partner violence: a global health perspective. CJNR (Canadian Journal of Nursing Research), 44(4), 36-54.

Hunnicutt, G. (2009). Varieties of patriarchy and violence against women: Resurrecting "patriarchy" as a theoretical tool. Violence against women, 15(5), 553-573. DOI: 10.1177/1077801208331246.

Jewkes, R. (2002). Intimate partner violence: causes and prevention. The lancet, 359(9315), 1423-1429. DOI: 10.1016/S0140-6736(02)08357-5.

Kim, J. C., Watts, C. H., Hargreaves, J. R., Ndhlovu, L. X., Phetla, G., Morison, L. A., ... \& Pronyk, P. (2007). Understanding the impact of a microfinance-based intervention on women's empowerment and the reduction of intimate partner violence in South Africa. American journal of public health, 97(10), 17941802. DOI: $10.2105 / \mathrm{AJPH} .2006 .095521$.

Kim, J. Y., \& Emery, C. (2003). Marital power, conflict, norm consensus, and marital violence in a nationally representative sample of Korean couples. Journal of Interpersonal Violence, 18(2), 197-219. https://doi.org/10.1177/0886260502238735.

Rahman, M., Nakamura, K., Seino, K., \& Kizuki, M. (2013). Does gender inequity increase the risk of intimate partner violence among women? Evidence from a national Bangladeshi sample. PLoS One, 8(12), e82423. https://doi.org/10.1371/journal.pone.0082423.

Semahegn, A., \& Mengistie, B. (2015). Domestic violence against women and associated factors in Ethiopia; systematic review. Reproductive health, 12(1), 78. https://doi.org/10.1186/s12978-015-0072-1.

Svec, J., \& Andic, T. (2018). Cooperative Decision-Making and Intimate Partner Violence in Peru. Population and development review, 44(1), 63-85. |https://doi.org/10.1111/padr.12127.

Tadesse, M., Teklie, H., Yazew, G., \& Gebreselassie, T. (2013). Women's Empowerment as a Determinant of Contraceptive use in Ethiopia Further Analysis of the 2011 Ethiopia Demographic and Health Survey. DHS Further Analysis Reports, 82.

United Nations (2013). Ethiopia: Leave No Women Behind. Retrieved from: http://www.unwomen.org/mdgf/B/Ethiopia_B.html. 
United Nations (2015). Transforming our world: the 2030 Agenda for Sustainable Development. Retrieved October 20, 2018 from: https://sustainabledevelopment.un.org/post2015/transformingourworld.

WHO (2002). World report on violence and health: summary. 2002. Geneva: World Health Organization.

WHO/London School of Hygiene and Tropical Medicine (2010). Preventing intimate partner and sexual violence against women. Taking action and generating evidence. Geneva, WHO, 2010. Retrieved from: http://apps.who.int/iris/bitstream/handle/10665/44350/9789241564007_eng.pdf;jsessionid=292C10567C6 575CD6FC2810825A9B86B?sequence=1.

WHO (2012). Understanding and addressing violence against women. Retrieved from: http://apps.who.int/iris/bitstream/handle/10665/77432/WHO_RHR_12.36_eng.pdf;jsessionid=C0B1C8194 D2FC0450532BED4D6783411? sequence $=1$.

WHO (2013). Global and regional estimates of violence against women: Prevalence and health effects of intimate partner violence and non-partner sexual violence. Retrieved from: http://apps.who.int/iris/bitstream/10665/85239/1/9789241564625_eng.pdf

WHO (2017). Media Center. Retrieved from: http://www.who.int/mediacentre/factsheets/fs239/en/.

World Economic Forum (2017). The Global Gender Gap Report 2017. Retrieved from: https://www.weforum.org/reports/the-global-gender-gap-report-2017.

Xu, X., Kerley, K. R., \& Sirisunyaluck, B. (2011). Understanding gender and domestic violence from a sample of married women in urban Thailand. Journal of Family Issues, 32(6), 791-819. https://doi.org/10.1177/0192513X10386306

Yodanis, C. L. (2004). Gender inequality, violence against women, and fear: A cross-national test of the feminist theory of violence against women. Journal of interpersonal violence, 19(6), 655-675. DOI: $10.1177 / 0886260504263868$. 